\title{
A New Radio-telemetering Apparatus for Measuring Raindrop-size
}

\author{
by \\ M. Fujiwara \\ Meteorological Research Institute \\ (Received April 26, 1954)
}

\begin{abstract}
The design and the construction of a new radio-telemetering instrument for measuring raindrops employing fiiter-paper as a sensing part for the raindrops are described. The reliabilities of the method of the new radio-telemetering instrument are discussed. It is shown that the instruments are suitable for accurate measurements especially for relatively smaller drops.
\end{abstract}

\section{Introduction}

In July and August 1952, a field work on the artificial production of precipitation was made in the Kanto district, Japan, as a joint study of the members of Meteorological Research Institute, Central Meteorological Observatory, Tokyo and Tokyo University, being sponsored by the Tokyo Electric Power Company. The present investigation was made as a part of the joint study and was reported preliminarily in Japanese in October 1952 [1].

The measurement of raindrop-size on the ground has been made by many investigators, Wiesenek [2], Bentley [3], Y. Takahashi [4], and I. Imai [5]. The measurement in the free atmosphere is badly needed in order to investigate the precipitation process. Theoretical evaluations of the growth of precipitation elements in the cloud have also been made by many investigators, and as a result the process of precipitation has become considerably clear, but we have at present scarcely any knowledge of the real nature of the distributions of raindrops in natural clouds. Recently, the rapid development of the technique of radar scanning has led to precipitation measurements by the echoes; this has in turn created a need of more complete knowledge of the distributions of raindrops in the cloud.

The measurement of raindrop-size distributions in the free atmosphere may be conducted on an aeroplane or with an adequate balloon-borne instrument. E. J. Smiтh [6] in Australia observed raindrops in non-freezing clouds by means of the former method. But the measurement on an aeroplane entails high cost and the high speed of the aeroplane causes various troubles.

In 1951 B. F. Cooper [7] developed a balloon-borne instrument for continuous measurements of raindrop-sizes.

The principal object of the research reported in this paper is the development of a new radio-telemetering balloon sounding apparatus with emphasis placed on relatively confident and convenient measurements of raindrop diameter and its concentration within clouds, expecting that the data obtained from flights with the new equipment will contribute to the understanding of atmospheric conditions 
and raindrop formation processes. The instrument has a phototube actinometer as an attachment in order to obtain some information on its location, i.e., whether it is flying in a cloud or not.

\section{Design of the new device and its principle}

For a design of the new type of radiosonde, it is very important to consider what telemetering system is the most advantageous. The measuring elements, which are related to the method of telemetering, are roughly classified into types such as variable capacitance, inductance, resistance and mechanical displacement. The variable resistance method is here adopted, using the leakage resistance of flecks on a filter-paper which acts as the sensing part for raindrops.

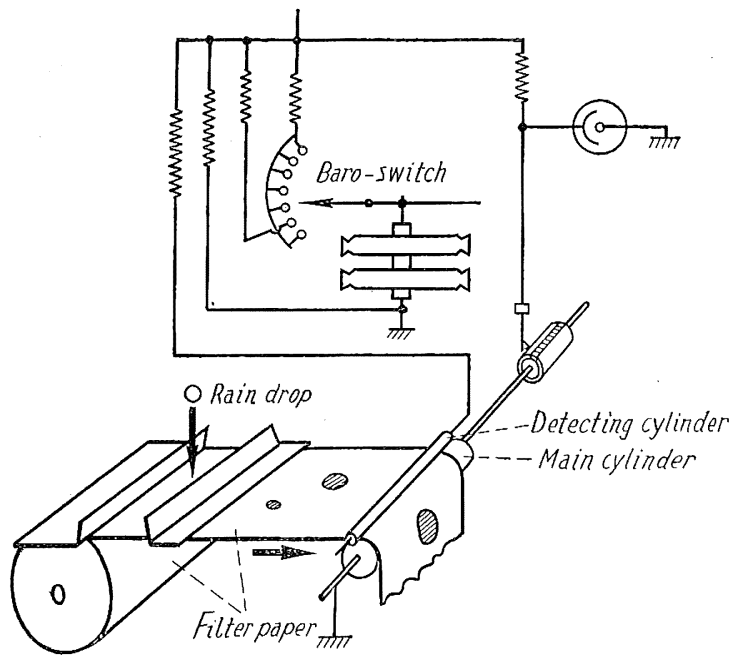

Fig. 1. Schematic diagram of measuring unit.

A schematic illustration of the devices is given in Fig. 1. This telemetering system is easily applied to a receiving set currently used in the routine aerological. observations in our country.

1) Raindrop measurement.

The well-known filter-paper method, in which a 2/3-power law is generally accepted, is more reliable than the momentum or light beam method for the measurement of raindrops, especially for relatively small drops. In order to detect the wet flecks, the filter-paper is run with a constant speed between two metal cylinders which constitute the electrodes in the $\mathrm{RC}$ circuit of a low frequency oscillator.

The spread flecks on the filter-paper are detected electrically, and the diameters of the flecks are measured from the length of their recorded signals.

Although pure water has a high specific resistance, a wet fleck of a natural raindrop presents the leakage resistance of several $10 \mathrm{~K} \Omega$. Rotating cylinders are electroplated. The driving speed of the filter-paper which is tape-shaped is from 2 to $2.5 \mathrm{~cm} / \mathrm{sec}$.

The time duration of the electrical leakage between the cylinders through. the flecks are proportional to both sizes of the flecks and the driving speeds: of the cylinders. The informations about the driving speeds are made by generated pulse-signals synchronized to the rotating cycles of the cylinder. The width of the filter-paper tape and its driving speed should be determined so as to diminish the probabilities of the fleck overlapping each other. The width should also be determined according to the rainfall intensity. The smaller the width of the paper, the larger the errors caused by the edge effects. In order to reduce the overlapping possibility, it is desirable to adjust the area of the inlet mouth of the raindrops, which limits the capture rate of drops.

2) Phototube actinometer.

The pulsed check-marks of the rotating cycles are generated in the form of 
negative pulses and are distinguished from the raindrop singnals. The pulse height depends upon the current in the phototube. When the photo-energy is very intense, i.e., when the instrument gets out of the cloud, the heights of the pulses will shrink to a constant level.

3) Pressure measurement.

In order to evaluate the location of the radiosonde, an aneroid switching system was employed with 7 contacts. The time during which the switch is in contact is from about 10 to 20 seconds and, therefore, very large compared with that of any other signal.

\section{Transmitting system}

The transmitter circuit is shown in Fig. 2 which is similar to the U.S. radiosonde. The used carrier frequency is of the band from $402 \mathrm{MC}$ to $408 \mathrm{MC}$.

A triod 2N3 which has been designed for radiosonde use in Japan is employed as the quenching oscillator tube. As Fig. 3 shows, laboratory tests indicate that it is a very stable oscillator tube for the purpose.

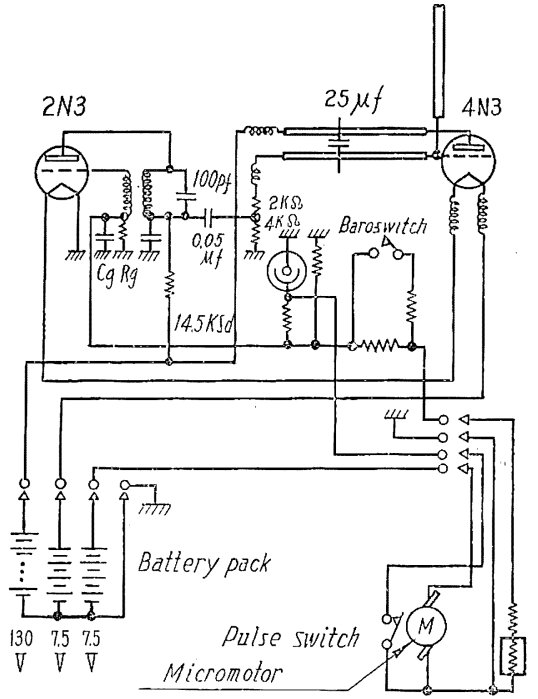

Fig. 2. Circuit diagram of telemetering unit.

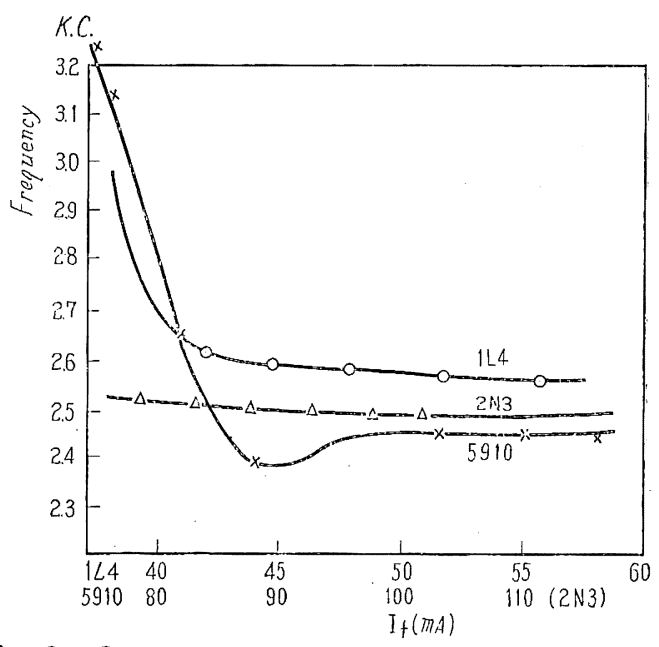

Fig. 3. Quenching frequency v.s. plate voltage relationships for oscillator tubes. It is shown that $2 \mathrm{~N} 3$ oscillates with the most constant frequency for the variation of plate voltages.

\section{Receiving set and recording equipment}

A block diagram of the receiving set is shown in Fig. 4. For this use, a receiving set used in the routine aerological observations is modified in some parts. A charge-discharge current method is adopted as a low frequency meter.

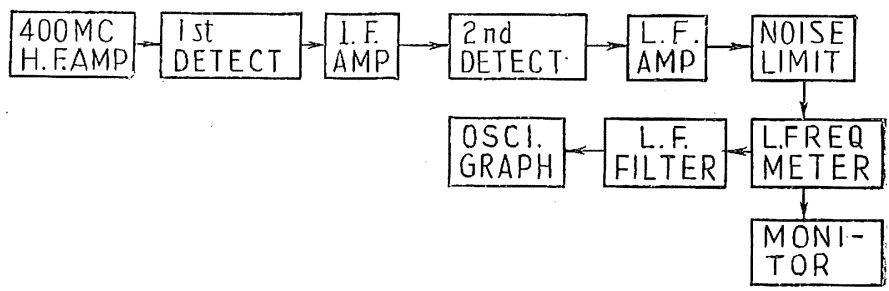

Fig. 4. Receiving set block diagram. 


\section{Considerations on the error factors}

The factors which affect considerably the validity of drop-size measurements to understanding the raindrop formation processes are 1) spreading rate and splashing of the raindrops on the filter-paper, 2) resolving power and capture efficiency, and 3) sampling error.

1) Spreading rate and splashing of raindrops on the filter-paper.

In this apparatus, the time interval from the fall of a raindrop on the filterpaper to the passage of its fleck between the two cylinders is required to be so long that the fleck may spread enough on the paper. Therefore the desirable minimum interval depends on the thickness of the filter-paper which is clesired to be as uniform and constant as possible.

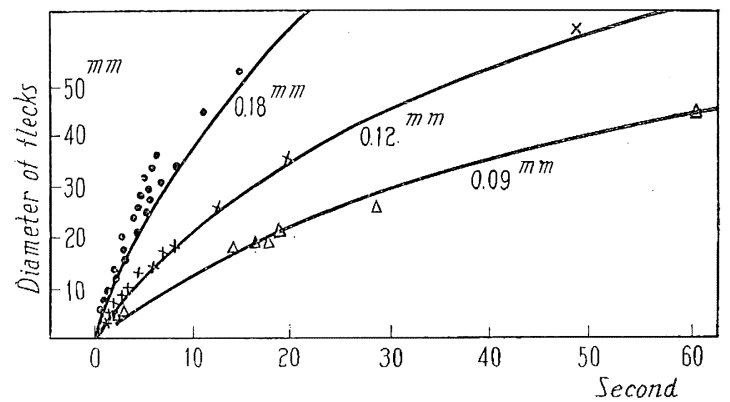

Fig. 5. Fleck spreading speed v.s. various filterpaper thickness.

Curves of the spreading rate are obtained as shown in Fig. 5 for three kinds of the filter-paper of $0.18,0.12$ and $0.09 \mathrm{~mm}$, in thickness. The ordinate indicates the maximum diameter of flecks spread enough, and the abscissa indicates the time needed for the spreading of flecks to the maximum sizes. It is clear that it takes longer for a fleck to spread sufficiently when the filter-paper is thinner. The curves indicate that in the region of larger drops more than twofold increase in the periods arises with decrease in the thickness of the filter-paper from $0.18 \mathrm{~mm}$ to $0.09 \mathrm{~mm}$.

Splashing is the most undesirable for our instrument, but even in the measurement of larger drops, the splashing errors could be acceptable values by the choice of the filter-paper of the suitable thickness. The star shape of the fleck produced at the moment of collision to filter-paper is gradually rounded by the diffusion of residual water at the center of the fleck. Therefore, it is needed that the filterpaper is very thin and is suspended freely. The typical splashing patterns are shown in Fig. 6. This favourable condition appears to be contrary to the demand mentioned above to reduce the spreading time. From these considerations the instruments are designed to employ the $0.1 \mathrm{~mm}$ filter-paper. The results of the laboratory tests indicate that the standard deviation of the error due to splashing will be not more than $5 \sim 7 \%$.

2) The measurable range of the raindrops.

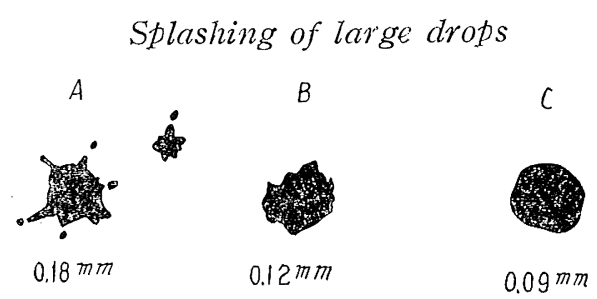

Fig. 6. Splashing patterns.

The upper limit of the measurable size depends upon splashing, as mentioned above. The lower limit is considered mainly governed by the capture efficiency of the smaller drops by the filter-paper, and also depends on the resolving power of the detection, which was examined to be about $0.1 \mathrm{~mm}$ in drop diameter.

The actual upper limit would be about $3.0 \mathrm{~mm}$ in drop diameter and in the region of larger size than this the accuracy would be somewhat deteriorated. 
3) Sampling error.

In any given instrument for measuring raindrop-size samling errors are inherent. It seems to be desirable to increase the capture rate of raindrops considering the sampling error. For this, the filter-paper must be considerably long, but the length of the paper is, in our case, restricted to some 30 meters for the measurement from the ground level up to about 6000 meter heights. This is due to the necessity of reducing the total loading weight of the balloon.

However much the capture rate of raindrops may be increased, it is difficult to infer or discuss the nature of the meteorological situations from one flight observation. Experiences of cloud observations show that a cloud generally consists of many cells which vary their locations successively. When radiosonde observations of raindrops are used to analyse the drop concentrations physically they should be made at least several times successively.

It has been shown by many investigators, J. O. Laws, D. A. Parsons [8] and A. C. BEst [9], that the volume distribution curves of exponential law closely fit the actual rain at the ground. The median diamter can be estimated from a comparatively small sample without large errors.

From these discussions the network and the frequency of the observational flights seem to be more essential for the sampling errors than the capture rate. It should be emphasized that the observations of the raindrop-sizes with such a telemetering instrument would profitably accompanied with a radio-direction-finding receiver and, if possible with radar PPI and RHI scopes, in order to obtain the knowledge of the flight path and its neighbouring precipitation cells. The new instrument is designed so as to transmit radio waves applicable to the rawin observations currently used in Japan.

\section{Test Flight}

The constructions and test flights as showen in Figs. 8 and 9 are made with considerable success.
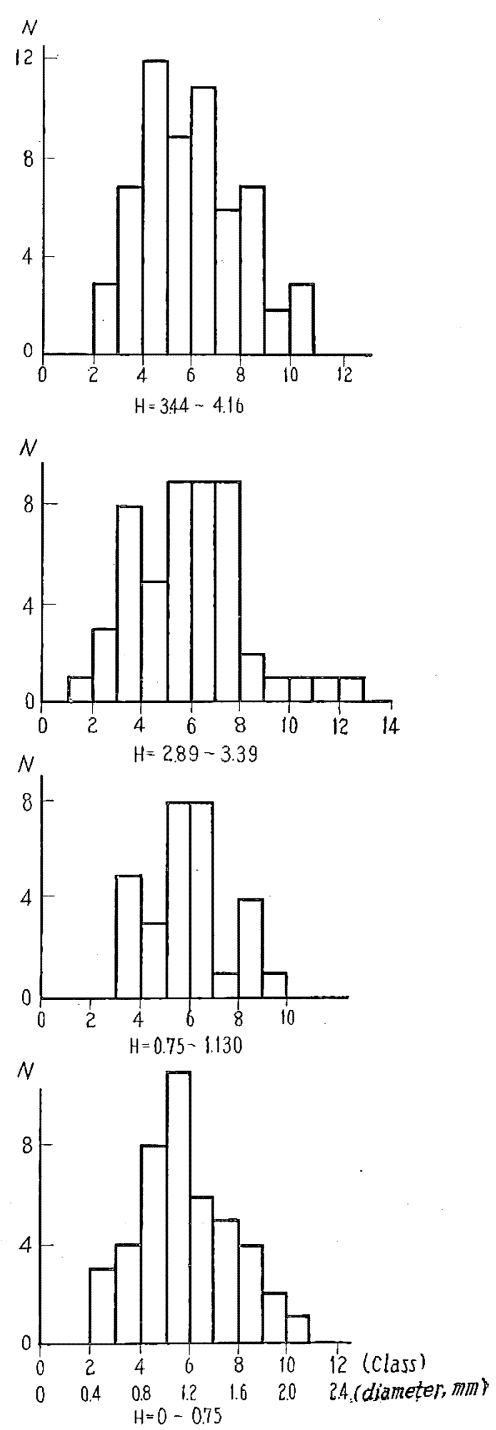

Fig. 7. An example of test flight. It was made in a shower-type. rainfall accompanied to a warm. front in October 1952 in Japan. The ordinate indicates counted numbers of drops in the size intervals. $H$ is an observed height interval in arbitrary unit equal to $\mathrm{Km}$ approximately. 


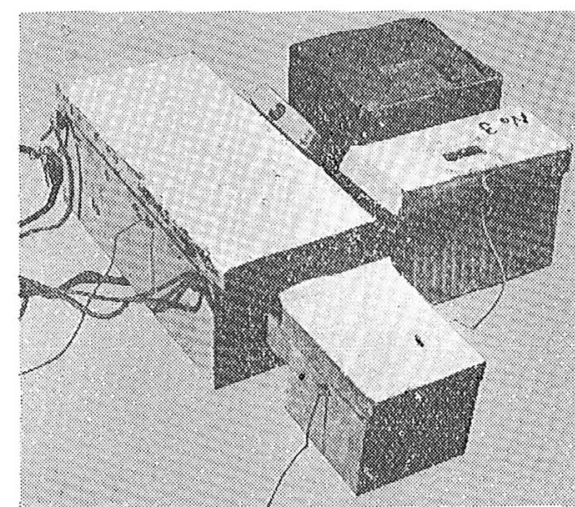

Fig. 8. Outersight of the instrument.

The left shows a measuring unit, and the right a telemetering unit which constitutes a battery pack (the largest box), an oscillator unit (the white box, on which a window for the phototube is seen) and a baro-switch box.

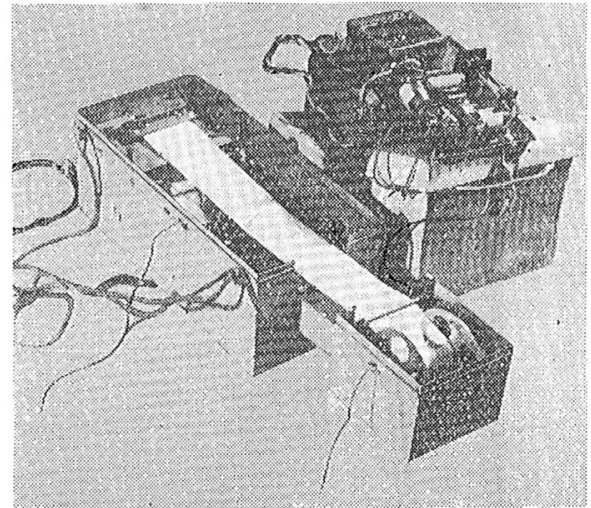

Fig. 9. Innersight of the instrument.

The white, tape-shaped filter-paper is seen in the measuring unit.

In the right-hand side, an oscillator unit and a baro-switch are shown.

Acknowledgements - The writer gratefully acknowledges the guidance and encouragement of Dr. I. IMAI, Mr. N. KodAira of M.R.I., and Prof. K. Isono of Tokyo University. He is likewise grateful to Dr. S. Syono of Tokyo Untversity and Mr. T. Yamaora, Director of the Aerological Observatory, Tateno, for their encouragement. He is also indebted to Mr. H. URABE of Kubota Meteorological Instrument Company for the cooperation and to Mr. M. Tohsha, Y. Toyama, I. ICHIMURA and Y. NABEShima for the cooperation in the construction, and test flights.

\section{References}

[1] Aerological Laboratory, Met. Res. Inst, 1951: On a Raindrop-radiosonde Used in the Field-Work of Artificial Rain Making (in Japanese).

[2] Wirsener, J., 1895: Beiträge zur Kenntnis des 'Tropischen Regens. Sitzber Akad. Wiss. Wien, Math-naturw. K1., 104, p. 1397.

[3] Bevrltiy, W.A., 1904: Studies of Raindrops and Raindrop Phenomenon.

[4] TAKaitashi, Y. 1935: Uber Regen und Regentropfen (Erste Mitteilung) Jour. Met. Soc. Japan. 13, p. 67.

[5] InaI, I., 19:40: Raindrop observations at the Foot of Mt. Akagi, Mem. Cen. Met. Obs. 21, p. 238.

[6] Sirth, J.E., 1951: Observation of Rain from Non-freezing Clouds. Quart. J. Roy. Meteor. Soc. 77, p. 33.

[7] COOPER, B. F., 1951: A Balloon-borne Instrument for Telemetering Raindrop-size Distribution and Rain Water Content of Cloud. Austral. J. Appl. Sci., , p. 43.

[8] Laws, J. O., and D. A. Parsons, 1943: The Relation of Raindrop-size to Intensity. Trans. Amer, Geophys. Un, 24, p. 452.

[9] Bfst, A. C., 1950: The Size Distribution of Raindrops. Quart. J. Roy. Meteor. Soc., 76, p. 16 . 